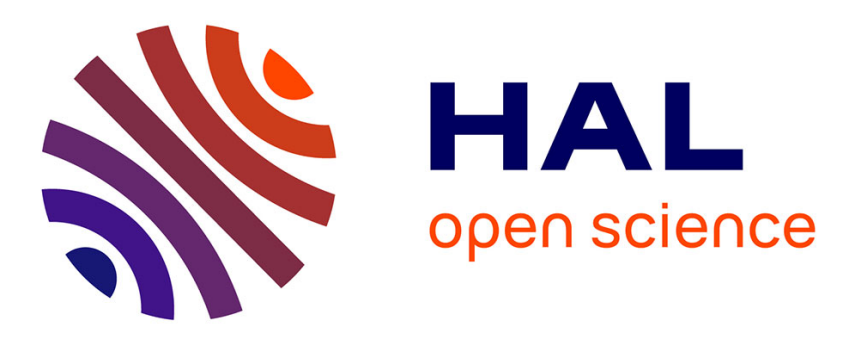

\title{
Novel "vibrating wire like" NEMS and MEMS structures for low temperature physics
}

Eddy Collin, J. Kofler, Jean-Savin Heron, Olivier Bourgeois, Yuriy M. Bunkov, Henri Godfrin

\section{- To cite this version:}

Eddy Collin, J. Kofler, Jean-Savin Heron, Olivier Bourgeois, Yuriy M. Bunkov, et al.. Novel "vibrating wire like" NEMS and MEMS structures for low temperature physics. Journal of Low Temperature Physics, 2010, 158 (3-4), pp.678-684. 10.1007/s10909-009-9960-5 . hal-00725962

\section{HAL Id: hal-00725962 https://hal.science/hal-00725962}

Submitted on 13 Jan 2014

HAL is a multi-disciplinary open access archive for the deposit and dissemination of scientific research documents, whether they are published or not. The documents may come from teaching and research institutions in France or abroad, or from public or private research centers.
L'archive ouverte pluridisciplinaire HAL, est destinée au dépôt et à la diffusion de documents scientifiques de niveau recherche, publiés ou non, émanant des établissements d'enseignement et de recherche français ou étrangers, des laboratoires publics ou privés. 


\title{
Novel "vibrating wire like" NEMS and MEMS structures for low temperature physics
}

\author{
E. Collin · J. Kofler · J.-S. Heron · O. Bourgeois · \\ Yu. M. Bunkov · H. Godfrin
}

15.06.2009

Keywords Micromechanics, nanomechanics, dissipation process, quantum fluid probes

Abstract Using microfabrication techniques, it has become possible to make mechanical devices with dimensions in the micro and even in the nano scale domain. Allied to low temperature techniques, these systems have opened a new path in physics with the ultimate goal of reaching the quantum nature of a macroscopic mechanical degree of freedom ${ }^{1}$. Within this field, materials research plays a significant role. It ranges from the fundamental nature of the dissipation mechanisms at the lowest temperatures, to the non-linear behavior of mechanical oscillators. We present experimental results on cantilever structures mimicking the well known "vibrating wire" technique, which present many advantages as far as the mechanical studies are concerned: the measurement is phase-resolved, they can be magnetomotive and electrostatically driven, and support extremely large displacements. Moreover, these devices can be advantageously used to study quantum fluids, making the link with conventional low temperature physics.

PACS numbers: 62.20.Dc, 62.40.+i, 81.40.Jj, 47.45.-n, 47.45.Ab

\section{Introduction}

Vibrating wires are a very common tool used in low temperature physics. A small piece of (usually superconducting) wire is immersed in a (quantum) fluid. From the damping and the frequency shift of the transverse resonance one can deduce the viscosity of the fluid ${ }^{2}$. This technique is effectively used to measure the lowest temperatures ever achieved directly inside superfluid ${ }^{3} \mathrm{He}^{3,4}$, and has been proposed to build ultra-sensitive low temperature astro-particle detectors ${ }^{5}$. The low temperature mechanics of these wires (in vaccum) has been extensively studied, especially with superconducting filaments ${ }^{6}$.

It has been realized with the first $4 \mathrm{~K}$ measurements of the Grenoble group ${ }^{7}$ that Silicon devices can replace advantageously this "classical" technique. Indeed, Aluminum-coated

Institut Néel

CNRS et Université Joseph Fourier,

BP 166, 38042 Grenoble Cedex 9, France

E-mail: eddy.collin@grenoble.cnrs.fr 


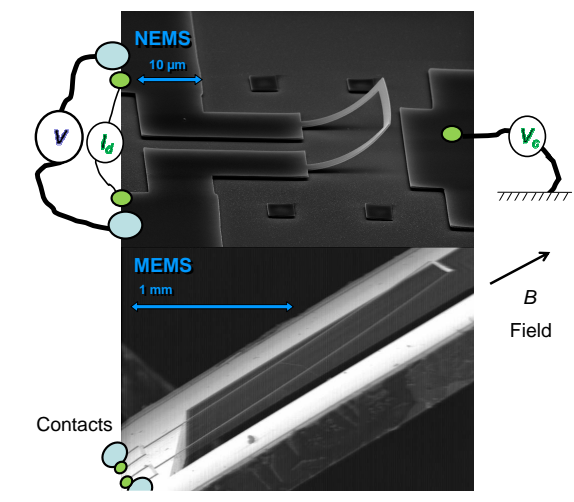

Fig. 1 (Color online) Two typical NbTi-coated structures mimicking the vibrating wire. Top: NEMS structure $200 \mathrm{~nm}$ thick, length $15 \mu \mathrm{m}$. The electrode on the right enables a capacitive coupling to the vibrating device. The metal layer is roughly $60 \mathrm{~nm}$ thick. Bottom: MEMS structure $5 \mu \mathrm{m}$ thick, length $1.85 \mathrm{~mm}$. The metal layer is roughly $150 \mathrm{~nm}$ thick. On both SEM pictures, one can see the curvature induced by the internal stress of the metallic layer created during the film growth ${ }^{11}$. This stress has an influence on the resonance position ${ }^{12}$. The drive and detection are also depicted, see text.

"goal-post" shaped $\mathrm{MEMS}^{(1)}$ were tested in the range $1 \mathrm{~K}-30 \mathrm{~K}$, and they displayed perfeclty suitable characteristics ${ }^{8}$. Their dynamical parameters can be very well determined and fit to the 1D (linear and non-linear) oscillator.

Moreover, the "vibrating wire like" geometry is perfectly well suited for low temperature mechanics on its own ${ }^{8}$. The cantilever-based geometry enables to study fundamentals of mechanics at the lowest temperatures, and can be efficiently used especially to characterize mechanical properties of metallic coatings. In Fig. 1 we present two of these structures: one of the size of a conventianal vibrating wire (mm long, $\mu \mathrm{m}$ thick), and another one roughly 100 times smaller ( $\mu \mathrm{m}$ long, $\mathrm{nm}$ thick) in the $\mathrm{NEMS}^{(2)}$ domain. The actuation and detection scheme enables a phase resolved measurement (which is not the case of most optical techniques ${ }^{9}$ ), and the deflections supported by these structures can be huge (while they are limited for a doubly-clamped beam ${ }^{10}$ ).

\section{Experimental details}

The fabrication method of the MEMS device has been reported in ${ }^{8,7}$. It consists in a first chemical etching $(\mathrm{KOH})$ releasing a thin membrane in a $\mathrm{Si}_{3} \mathrm{~N}_{4}$ protected Silicon chip. The Nitride is then removed, and after a standard optical lithography we use Reactive Ion Etching to pattern the goal-post shape on it.

The NEMS device is obtained from SOI wafers ${ }^{13}$. After an e-beam lithography, the structure is patterned by means of Reactive Ion Etching. The $200 \mathrm{~nm}$ oxyde is then removed by a chemical vapour HF etching, releasing the device.

The excitation and detection scheme is the standard vibrating wire technique (see Fig. 1). A sinusoidal current $I_{d} \cos (\omega t)$ is driven in the structure which lies in a static magnetic field $B$ parallel to the chip surface. A magnetomotive force $I_{d} l B$ appears pulling the structure

\footnotetext{
1 MEMS: micro-electro-mechanical-systems
}

2 NEMS: nano-electro-mechanical-systems. 


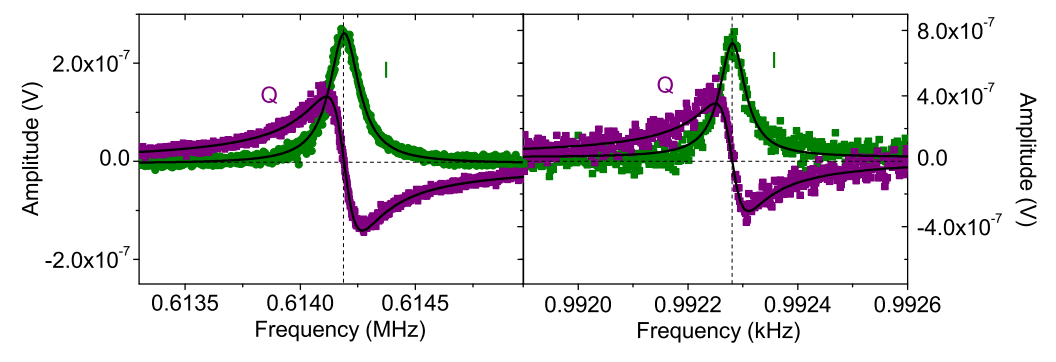

Fig. 2 (Color online) Linear resonance obtained for the transverse mode, at $4.2 \mathrm{~K}$ in vacuum. I and Q stand for phase and quadrature components respectively. Left: NEMS response with a current of $0.4 \mu \mathrm{A}$ in a field of $540 \mathrm{mT}$. Right: MEMS response with a current of $0.3 \mu \mathrm{A}$ in a field of $14 \mathrm{mT}$. The lines are Lorentzian fits.

out of the plane ( $l$ is the length of the pad). As the angular frequency $\omega$ is swept through the mode resonance $\omega_{0}$ the structure starts to vibrate and an induced voltage $V=l B d x / d t$ appears at its ends (with $x$ the top end displacement). It is detected with a lock-in amplifier giving acess to phase (I) and quadrature (Q). For the NEMS structure, a capacitor has been added to the design, enabling a voltage drive $V_{c}$.

Two typical resonance curves are shown in Fig. 2, in the superconducting state of the $\mathrm{NbTi}$ coating. The phase and quadrature components of the lines are perfectly Lorentzian, with a $Q$ value of 4000 for the NEMS and 17000 for the MEMS respectively. While not particularly large, these values are within the literature ${ }^{14,15}$. Note that Aluminum coated vibrating wire MEMS $^{8}$ can reach $Q$ values of the order of $0.510^{6}$.

\section{Results}

Keeping the aspect ratios roughly constant, it is possible to compare directly the impact of the size $(\mu \mathrm{m}$ to $\mathrm{nm})$ on the mechanical parameters: the internal friction mechanisms ${ }^{16,17}$, the strength and elasticity of the metallic films deposited ${ }^{18}$, the non-linear characteristics of the cantilevers and the superconducting-induced effects. In particular the damping mechanisms limiting the $Q$ factors at low and ultra-low temperatures deserve to be understood ${ }^{19,20}$. In the present article, we shall discuss only a limited amount of points which we consider as most relevant. The whole of the experiment shall be published elsewere.

On Fig. 3 we show the characteristic displacement-force curve of the NEMS device, in the normal state. For the largest excitations, heating effects have been seen ${ }^{(3)}$ and quantitatively measured ( $\delta T<2 \mathrm{~K}$ for the $10 \mathrm{~K}$ data of Fig. 3). For our largest displacements, the NEMS structure started to behave non-linearly. The dynamic range explored is even wider than for our MEMS structures, but results are similar (Fig. 5 of ref. ${ }^{8}$ ).

The normal-state linear and non-linear behaviour of our MEMS structures is fairly well understood $^{8}$. The non-linearity is of geometrical origin, leading to a frequency shift of the order or $\delta f=+0.1 f_{0} x^{2} / h^{2}$ ( $h$ is the length of the cantilever). Thus the non-linearity is visible as soon as the shift is of the order of the linewidth $\Delta f$, which leads to the simple

\footnotetext{
3 The line shifts and broadens, while keeping a Lorentz shape.
} 


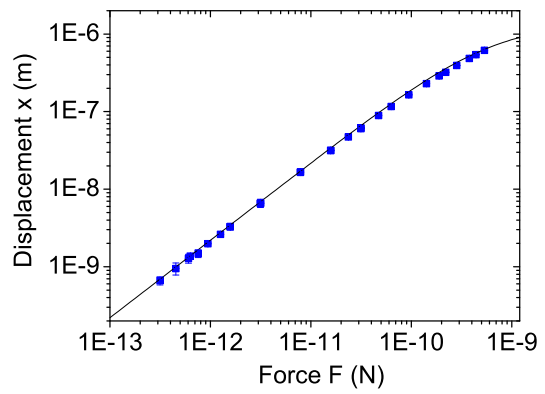

Fig. 3 (Color online) Displacement versus force curve for the NEMS structure, measured in vacuum at $10 \mathrm{~K}$ (normal state of the NbTi coating). The line is a fit enabling to "weigh" the device: we have typically $k \approx$ $1.5 \mathrm{~N} / \mathrm{m}$ (for the pair of cantilevers) and $m \approx 1.10^{-13} \mathrm{~kg}$ (for the vibrating mass). Note the dynamic range explored by the device. For an equivalent MEMS device, see Fig. 5 of ref. ${ }^{8}$.

relation:

$$
(x / h)^{2}=10 / Q
$$

from which we obtain numbers in the $10 \mu \mathrm{m}-20 \mu \mathrm{m}$ range for $x$, that is of the order of $0.5 \%$ to $1 \%$ of $h$. When the non-linearity appears, the shape of the resonance becomes triangular with a frequency pulled upwards. At some critical excitation, the response gets bivalued $^{21}$.

In Fig. 3 the displacement of the end part of the NEMS reaches about $4 \%$ of its length $h$, and indeed the line starts to become non-linear. However, the non-linearity is very small and has the opposite sign (it is pulled downwards), which deserves to be fully understood. In particular, the non-linear coefficient extracted from direct line fits is strain-dependent, and future experiments should clarify also its temperature dependence. It certainly is materialdependent (i.e. the NbTi layer) and perhaps linked to the huge distortion of the structure.

Moreover, a measured linear regime extending over 2 orders of magnitude is remarkable, and illustrates also the sensitivity of the setup. Indeed, the linear dynamic range is a figure of merit used in signal processing that can be applied to MEMS/NEMS in order to compare and characterize them ${ }^{22}$.

On Fig. 4 we demonstrate the capacitive coupling to the device. To do so, we show our ability to tune the resonant frequency with an applied DC voltage $V_{c}$ on the electrode. This voltage adds up a restoring force which in turn produces a restoring spring $\propto V_{c}^{2}$.

On Fig. 5 we illustrate the effects of superconductivity on the mechanical parameters (here, the frequency). Indeed, at low enough fields the frequency of the oscillator shifts up quadratically ${ }^{23}$ with $B$. This effect had been reported already for our MEMS structure ${ }^{24}$ down to $1 \mathrm{mT}$. It is easily understood as the 'pure' Meissner effect expelling the field lines from the superconductor, or an 'effective' Meissner effect obtained with strongly pinned vortices in the metal. In both cases the field lines distorsion gives rise to an additional spring constant (through the field lines energy gradient) leading to $f=\sqrt{f_{0}^{2}+a B^{2}}$. The data are taken at low drives where the damping seems to be field and displacement independent. For stronger fields and drives, the frequency dependence is more complex, with additional dissipation occuring, certainly through vortex dynamics. The quadratic dependence factor $a$ has been extracted and is plotted versus temperature in Fig. 5. It clearly reflects the supercon- 


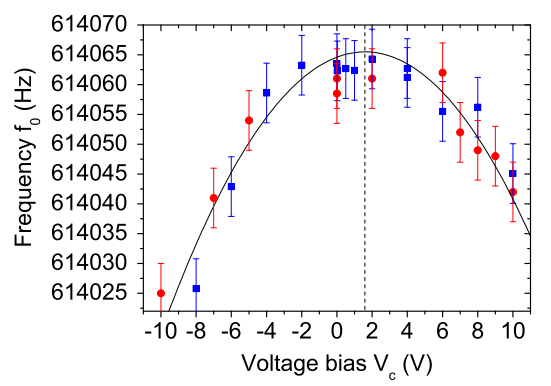

Fig. 4 (Color online) Tuning of the frequency by means of the capacitive coupling. A static voltage is applied $V_{c}$ on the electrode while the resonance is swept with the standard technique. Data taken at $4.2 \mathrm{~K}$ (blue squares) and $10 \mathrm{~K}$ (red circles, shifted on the $y$ axis), with low fields and small drives. The line is a quadratic fit, giving a coupling of $-0.35 \mathrm{~Hz} / \mathrm{V}^{2}$.

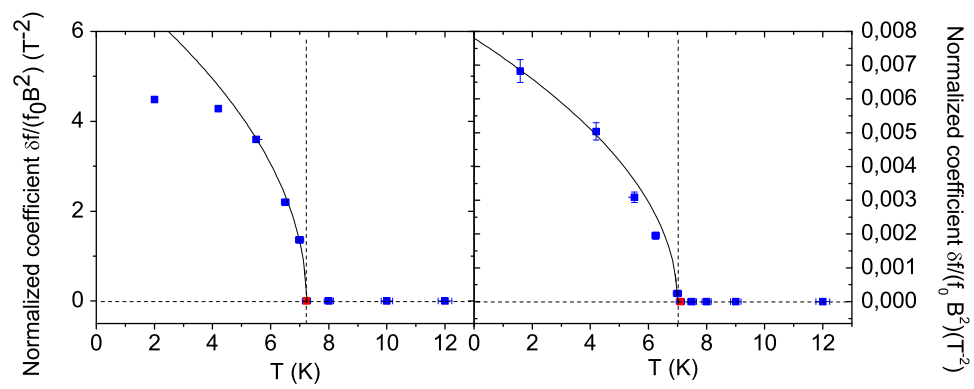

Fig. 5 (Color online) Quadratic factor extracted from the field induced frequency shift at small fields, as a function of temperature $T$. On the left: NEMS device. On the right: MEMS device. For both the $\mathrm{NbTi} T_{c}$ was about $7.3 \mathrm{~K}$. The line is a $\left(T_{c}-T\right)^{0.5}$ guide (see text).

ducting transition with a power law dependance of $T_{c}-T$ close to $T_{c}$ and a saturation at low temperatures. A fine understanding of the effect should lead to a quantitative analysis giving access, for instance, to the London penetration length $\lambda(T)$.

\section{Conclusions}

In this paper we presented new "vibrating wire like" MEMS and NEMS devices for low temperature studies. The mechanical resonance of these structures is obtained via the magnetomotive technique, furnishing a phase-resolved signal. The geometry allows for very strong displacements, and we can follow the vibration amplitude up to the non-linear regime of the MEMS and NEMS cantilevers.

Moreover, this technique is a convenient tool to study mechanics (for instance elasticity) of thin metallic films (both normal and superconducting), and could be used for viscometry in quantum fluids down to the $100 \mathrm{~nm}$ scale. 


\section{References}

1. M. D. LaHaye, O. Buu, B. Camarota, K. C. Schwab, Science 304, p. 74 (2004).

2. M.A. Black, H.E. Hall, K. Thomson, Proc. 10th Int. Conf. on Low Temperature Physics, p. 174 (1966); M.A. Black, H.E. Hall, K. Thomson, J. Phys. C: Solid St. Phys. 4, p. 129 (1971).

3. A.M. Guénault, V. Keith, C.J. Kennedy, S.G. Mussett and G.R. Pickett, J. of Low Temp. Phys. 62, p. 511 (1986).

4. C. Bäuerle, Yu.M. Bunkov, S.N. Fisher and H. Godfrin, Phys. Rev. B 57, p. 14381 (1998); C.B. Winkelmann, E. Collin, Yu.M. Bunkov, H. Godfrin, J. of Low Temp. Phys. 135, p. 3 (2004).

5. C.B. Winkelmann, J. Elbs, Yu.M. Bunkov, E. Collin, H. Godfrin, M. Krusius, Nuclear Instr. and Methods in Physics Research A 574, p. 264 (2007).

6. R. König, P. Esquinazi and F. Pobell, J. of Low Temp. Phys. 90, p. 55 (1993).

7. S. Triqueneaux, E. Collin, D. J. Cousins, T. Fournier, C. Bäuerle, Yu. M. Bunkov and H. Godfrin, Physica B 284, p. 2141 (2000).

8. E. Collin, Laure Filleau, Thierry Fournier, Yuriy M. Bunkov and Henri Godfrin, J. of Low Temp. Phys. 150, no 5-6, p. 739 (2008).

9. Dustin W. Carr, S. Evoy, L. Sekaric, H. G. Craighead, and J. M. Parpia, Appl. Phys. Lett. 75, p. 920 (1999).

10. Guiti Zolfagharkhani, Alexei Gaidarzhy, Seung-Bo Shim, Robert L. Badzey, and Pritiraj Mohanty, Phys. Rev. B 72, 224101 (2005).

11. Michel Godin, Vincent Tabard-Cossa, and Peter Grütter, Peter Williams, Appl. Phys. Lett. 79, p. 551 (2001).

12. Andrew W. McFarland, Mark A. Poggi, Margaret J. Doyle, and Lawrence A. Bottomley, Jonathan S. Colton, Appl. Phys. Lett. 87, 053505 (2005).

13. J.-S. Heron, T. Fournier, N. Mingo and O. Bourgeois, Nano Letters 9, 1861 (2009).

14. R. E. Mihailovich and J. M. Parpia, Phys. Rev. Lett. 68, p. 3052 (1992).

15. P. Mohanty, D.A. Harrington, K.L. Ekinci, Y.T. Yang, M.J. Murphy, and M.L. Roukes, Phys. Rev. B 66, 085416 (2002).

16. C. Seoánez and F. Guinea, A. H. Castro Neto, Phys. Rev. B 77, p. 125107 (2008).

17. R. Lifshitz and M. L. Roukes, Phys. Rev. B 61, p. 5600 (2000).

18. M. A. Haque, and M. T. A. Saif, PNAS 101, p. 6335 (2005).

19. A. D. Fefferman, R. O. Pohl, A. T. Zehnder, and J. M. Parpia, Phys. Rev. Lett. 100, p. 195501 (2008).

20. J. Classen, T. Burkert, C. Enss, and S. Hunklinger, Phys. Rev. Lett. 84, p. 2176 (2000).

21. L.D. Landau and E.M. Lifshitz, Mechanics, third ed. Elsevier Science Ltd. (1976).

22. K. L. Ekinci, M. L. Roukes, Rev. of Sci. Instr. 76, 061101 (2005); H. W. Ch. Postma, I. Kozinsky, A. Husain, and M. L. Roukes, Appl. Phys. Lett. 86, 223105 (2005).

23. J. Kober, A. Gupta, P. Esquinazi, H.F. Braun, Phys. Rev. Lett., 66, p. 2507 (1991).

24. Poster session, "Superconductor coated electro-mechanical systems for low and ultralow temperature physics", E. Collin, Y. Bilbao-Zarraga, Yu. M. Bunkov, H. Godfrin, ULT 2008 conference frontiers of low temperature physics, Royal Holloway University of London, England, 14-17 August 2008. 